PROCEEDINGS OF THE

AMERICAN MATHEMATICAL SOCIETY

Volume 137, Number 11, November 2009, Pages 3569-3574

S 0002-9939(09)10012-6

Article electronically published on June 15, 2009

\title{
LOWER BOUNDS FOR MOMENTS OF AUTOMORPHIC $L$-FUNCTIONS OVER SHORT INTERVALS
}

\author{
GUANGHUA JI
}

(Communicated by Wen-Ching Winnie Li)

\begin{abstract}
Let $L(s, \pi)$ be the principal $L$-function attached to an irreducible unitary cuspidal automorphic representation $\pi$ of $G L_{m}\left(\mathbb{A}_{\mathbb{Q}}\right)$. The aim of the paper is to give a simple method to show the lower bounds of mean value for automorphic $L$-functions over short intervals.
\end{abstract}

\section{INTRODUCTION}

An important problem in analytic number theory is to estimate the moments

$$
I_{k}(T)=\int_{T}^{2 T}\left|L\left(\frac{1}{2}+i t, \pi\right)\right|^{2 k} \mathrm{~d} t
$$

for all kinds of $L$-functions $L(s, \pi)$. For the Riemann zeta-function $\zeta(s)$, a conjecture states that the $2 k$-th moment should be asymptotic to $C_{k} T(\log T)^{k^{2}}$ for a positive constant $C_{k}$, where $k$ is a positive real number. The correct upper bound of $I_{k}(T)$ for $\zeta(s)$ is known only for $k \leq 2$, while the correct lower bound is proved for all rational $k \geq 0$,

$$
I_{k}(T) \gg T(\log T)^{k^{2}} \text {. }
$$

Actually, this latter result was proved by Ramachandra [11 for all positive integers $k$, by Heath-Brown 4 for all positive rational numbers $k$, and under the Riemann Hypothesis by Ramachandra [10 for all positive real numbers $k$. In 1 ] the authors propose conjectures for the full asymptotics of the moments of general $L$-functions. In particular, the paper provides conjectures for the moments of the Riemann zeta-function, the family of primitive Dirichlet $L$-functions, quadratic twists of $L$-functions, and automorphic $L$-functions attached to cusp forms.

Let $\pi$ be an irreducible unitary cuspidal automorphic representation of $G L_{m}\left(\mathbb{A}_{\mathbb{Q}}\right)$ and $s=\sigma+i t \in \mathbb{C}$. Then the principal $L$-function [3] attached to $\pi$ is given by Euler products of local factors for $\sigma>1$,

$$
\begin{aligned}
L(s, \pi) & =\prod_{p<\infty} L\left(s, \pi_{p}\right) \\
& =\sum_{n=1}^{\infty} \frac{a_{\pi}(n)}{n^{s}},
\end{aligned}
$$

Received by the editors October 20, 2008.

2000 Mathematics Subject Classification. Primary 11F66, 11M26, 11M41.

(C)2009 American Mathematical Society Reverts to public domain 28 years from publication 
where

$$
L\left(s, \pi_{p}\right)=\prod_{j=1}^{m}\left(1-\alpha_{\pi}(p, j) p^{-s}\right)^{-1}
$$

with $\left\{\alpha_{\pi}(p, j): 1 \leq j \leq m\right\}$ being complex Satake parameters of $\pi$ at the finite place $\pi_{p}$ according to the local Langlands correspondence. If $p$ is unramified, its local $L$-factor is of the form $L\left(s, \pi_{p}\right)=P_{p}\left(p^{-s}\right)^{-1}$, where $P_{p}(x)$ is a polynomial of degree at most $m$ and $P_{v}(0)=1$. We can write the local factors at ramified places $v$ in the same form with the convention that some of the $\alpha_{\pi}(p, j)$ may be zero.

In this paper, we give a simple method to obtain lower bounds for integral moments of the principal $L$-function $L(s, \pi)$ over short intervals. Our main theorem is as follows.

Theorem 1.1. Let $\pi$ be an irreducible cuspidal automorphic representation of $G L_{m}\left(\mathbb{A}_{\mathbb{Q}}\right)$ and let $k$ be any positive real number. Then uniformly in $\sigma$,

$$
\int_{T}^{T+H}|L(\sigma+i t, \pi)|^{k} \mathrm{~d} t \gg H
$$

for all $T \geq T_{0}$ for some sufficiently large $T_{0}, \sigma \geq 1 / 2$, and $T \geq H \geq \log ^{1+\epsilon} T$ with any $\epsilon>0$.

\section{Automorphic $L$-Functions And some lemmas}

Let $\pi$ be an irreducible cuspidal automorphic representation of $G L_{m}\left(\mathbb{A}_{\mathbb{Q}}\right)$. Denote the complete $L$-function by

$$
\Lambda(s, \pi)=q^{\frac{s}{2}} L\left(s, \pi_{\infty}\right) L(s, \pi),
$$

where $q$ is an integer called the arithmetic conductor of $\pi$ and

$$
L\left(s, \pi_{\infty}\right)=\prod_{j=1}^{m} \Gamma_{\mathbb{R}}\left(s+\mu_{j}\right) .
$$

Here $\Gamma_{\mathbb{R}}(s)=\pi^{s / 2} \Gamma\left(\frac{s}{2}\right)$, and $\left\{\mu_{j}: 1 \leq j \leq m\right\}$ are local complex parameters of $\pi$ at the infinite place $\infty$.

The following statements collect together analytic facts about principal $L$-functions which we will use for our proofs.

(A1) The Dirichlet series

$$
L(s, \pi)=\sum_{n=1}^{\infty} \frac{a_{\pi}(n)}{n^{s}}
$$

converges absolutely in the half-plane $\Re s>1$, and we have (see [9])

$$
\sum_{n \leq X}\left|a_{\pi}(n)\right|^{2} \ll X^{1+\epsilon} .
$$

(A2) The complete $L$-function $\Lambda(s, \pi)$ has an analytic continuation to the whole complex plane and satisfies the functional equation [3]

$$
\Lambda(1-s, \tilde{\pi})=\varepsilon(\pi) \Lambda(s, \pi)
$$

where $\varepsilon(\pi)$, a complex number of modulus 1 , is the root number, $\tilde{\pi}$ is the contragredient representation of $\pi$, and $L_{v}(s, \tilde{\pi})=\overline{L_{v}(\bar{s}, \pi)}$ for any place $v$. 
(A3) $\Lambda(s, \pi)$ is an entire function of order one, bounded in vertical strips with finite width with exponential decay as $|\Im s| \rightarrow \infty$; see [2], [3].

(A4) The zeros of $\Lambda(s, \pi)$, that is, the nontrivial zeros of $L(s, \pi)$, lie in the open critical strip $0<\Re s<1$; see 6. In particular, $L(s, \pi)$ is nonvanishing in the half-plane $\Re s \geq 1$.

(A5) Bounds toward the generalized Ramanujan conjecture, 8, 12, are

$$
\begin{aligned}
& \left|\alpha_{\pi}(p, j)\right| \leq p^{\frac{1}{2}-\frac{1}{m^{2}+1}} \quad \text { if } \pi \text { is unramified at } p, \\
& \left|\Re \mu_{j}\right| \leq \frac{1}{2}-\frac{1}{m^{2}+1} \quad \text { if } \pi \text { is unramified at } \infty .
\end{aligned}
$$

We also need the some lemmas. The following result of Littlewood establishes the connection between zeros of an analytic function and its mean-value estimates.

Lemma 2.1. Let $\phi(s)$ be analytic and nonzero on the rectangle $\mathcal{D}$ with vertices $\alpha$, $\beta, \alpha+i T$, and $\beta+i T$, where $\alpha<\beta$. Then

$$
\begin{aligned}
2 \pi \sum_{\rho \in \mathcal{D}} \operatorname{Dist}(\rho)= & \int_{0}^{T} \log |\phi(\alpha+i t)| \mathrm{d} t-\int_{0}^{T} \log |\phi(\beta+i t)| \mathrm{d} t \\
& +\int_{\alpha}^{\beta} \arg \phi(\sigma+i T) \mathrm{d} \sigma-\int_{\alpha}^{\beta} \arg \phi(\sigma) \mathrm{d} \sigma,
\end{aligned}
$$

where the sum runs over the zeros $\rho$ of $\phi(s)$ in $\mathcal{D}$, and $\operatorname{Dist}(\rho)$ is the distance from $\rho$ to the left edge of the rectangle.

Proof. This is a revised version of the classical Littlewood lemma. See Titchmarsh 13. Sections 9.9 and 9.15 .

Lemma 2.2. (1) Let $N(T)$ be the number of nontrivial zeros $\rho=\beta+i \gamma$ of $L(s, \pi)$ such that $0<\beta<1$ and $0<\gamma \leq T$. Then

$$
N(T+1)-N(T) \ll \log T .
$$

(2) For any $s=\sigma+i$ in the strip $-2 \leq \sigma \leq 2,|t| \geq t_{0}$, where $t_{0}$ is a fixed positive constant, we have

$$
\frac{L^{\prime}}{L}(s, \pi)=\sum_{|t-\gamma| \leq 1} \frac{1}{s-\rho}+O(\log |t|) .
$$

(3) With the same notation as in (2), we have

$$
\log L(s, \pi)=\sum_{|t-\gamma| \leq 1} \log (s-\rho)+O(\log |t|) .
$$

Proof. For proofs of (1) and (2), see Iwaniec and Kowalski [5, Proposition 5.7, or Liu and Ye [7, Lemma 4.3. We integrate (2.4) along the straight line from $s=\sigma+i t$ to $2+i t$. By (2.3), if $t$ is not the ordinate of a zero, then

$$
\begin{aligned}
\log L(s, \pi)= & \log L(2+i t, \pi)+\sum_{|t-\gamma| \leq 1} \log (s-\rho) \\
& -\sum_{|t-\gamma| \leq 1} \log (2+i t-\rho)+O(\log |t|) \\
= & \sum_{|t-\gamma| \leq 1} \log (s-\rho)+O(\log |t|) .
\end{aligned}
$$


This proves the lemma.

\section{Proof of Theorem 1.1}

We apply Lemma 2.2 with $\phi(s)=L(s, \pi), \beta=2$, and $\alpha=\sigma_{0}$ for some $1 / 2 \leq$ $\sigma_{0} \leq 1$. If $T$ is not the ordinate of a zero, then

$$
\begin{aligned}
2 \pi \sum_{\rho \in \mathcal{D}} \operatorname{Dist}(\rho)= & \int_{0}^{T} \log \left|L\left(\sigma_{0}+i t, \pi\right)\right| \mathrm{d} t-\int_{0}^{T} \log |L(2+i t, \pi)| \mathrm{d} t \\
& +\int_{\sigma_{0}}^{2} \arg L(\sigma+i T, \pi) \mathrm{d} \sigma+K\left(\sigma_{0}\right),
\end{aligned}
$$

where $K\left(\sigma_{0}\right)$ is independent of $T$.

Lemma 3.1. If $1 / 2 \leq \sigma \leq 1$ and $T$ is sufficiently large, then

$$
2 \pi \sum_{\rho \in \mathcal{D}} \operatorname{Dist}(\rho)=\int_{0}^{T} \log |L(\sigma+i t, \pi)| \mathrm{d} t+O(\log T),
$$

where $\mathcal{D}$ is the rectangle with vertices $\sigma, 2, \sigma+i T$, and $2+i T$.

Proof. We define

$$
\Lambda_{\pi}(n)=\sum_{j=1}^{m} \alpha_{\pi}^{\ell}(p, j) \log p
$$

if $n=p^{\ell}$ with $\ell$ being a positive integer and 0 otherwise. By (A5), we have

$$
\Lambda_{\pi}(n) \ll n^{\frac{1}{2}-\frac{1}{m^{2}+1}} \log n .
$$

For $\sigma>1$, we deduce

$$
\begin{aligned}
\log L(s, \pi) & =\sum_{p} \sum_{n=1}^{\infty} \frac{\Lambda_{\pi}\left(p^{n}\right)}{\left(\log p^{n}\right) p^{n s}} \\
& =\sum_{n=2}^{\infty} \frac{\Lambda_{\pi}(n)}{(\log n) n^{s}} .
\end{aligned}
$$

Therefore,

$$
\begin{aligned}
\int_{0}^{T} \log |L(2+i t, \pi)| \mathrm{d} t & =\Re\left(\int_{0}^{T} \log L(2+i t, \pi) \mathrm{d} t\right) \\
& =\Re\left(\sum_{n=2}^{\infty} \frac{\Lambda_{\pi}(n)}{(\log n) n^{2}} \frac{n^{-i T}-1}{-i \log n}\right) \\
& \ll 1 .
\end{aligned}
$$

Since $|\arg (\sigma+i T-\rho)| \leq \pi$ for $1 / 2 \leq \sigma \leq 1$, we deduce by (2.5) and then (2.3) that

$$
\begin{aligned}
\arg L(\sigma+i T, \pi) & =\Im(\log L(\sigma+i T, \pi)) \\
& =\sum_{|T-\gamma| \leq 1} \arg (\sigma+i T-\rho)+O(\log T) \\
& \ll \log T .
\end{aligned}
$$


The lemma immediately follows from (3.1), (3.3), and (3.4).

Proof of Theorem 1.1. According to (3.2), we get

$$
2 \pi \sum_{\rho \in \mathcal{D}} \operatorname{Dist}(\rho)=\int_{T}^{T+H} \log |L(\sigma+i t, \pi)| \mathrm{d} t+O(\log T),
$$

where $H=\log ^{1+\epsilon} T$ with any fixed $\epsilon>0$ and $\mathcal{D}$ is the rectangle with vertices $\sigma+i T$, $2+i T, \sigma+i(T+H)$, and $2+i(T+H)$. The left-hand side of (3.5) is nonnegative. Hence, there exists a constant $C>0$ such that

$$
\int_{T}^{T+H} \log |L(\sigma+i t, \pi)| \mathrm{d} t \geq-C \log T
$$

where $1 / 2 \leq \sigma \leq 1$. Now recall that if $a<b, f(t) \geq 0$ for $a \leq t \leq b$ and $f(t)$ is a continuous real function on the interval $[a, b]$, then

$$
\frac{1}{b-a} \int_{a}^{b} \log f(t) \mathrm{d} t \leq \log \left(\frac{1}{b-a} \int_{a}^{b} f(t) \mathrm{d} t\right),
$$

which is an easy consequence of the inequality between the arithmetic and geometric means of nonnegative numbers. Taking $f(t)=|\log L(\sigma+i t, \pi)|^{k}$ for any fixed positive real number $k$, we get

$$
\begin{aligned}
\log \left(\frac{1}{H} \int_{T}^{T+H}|L(\sigma+i t, \pi)|^{k} \mathrm{~d} t\right) & \geq \frac{1}{H} \int_{T}^{T+H} \log |L(\sigma+i t, \pi)|^{k} \mathrm{~d} t \\
& =\frac{k}{H} \int_{T}^{T+H} \log |L(\sigma+i t, \pi)| \mathrm{d} t \\
& \geq-C k \frac{\log T}{H} .
\end{aligned}
$$

Hence,

$$
\begin{aligned}
\int_{T}^{T+H}|L(\sigma+i t, \pi)|^{k} \mathrm{~d} t & \geq H \exp \left(-C k \frac{\log T}{H}\right) \\
& =H\left(1+O\left(\frac{\log T}{H}\right)\right) \\
& \gg H,
\end{aligned}
$$

for $\log ^{1+\epsilon} T \leq H \leq T$ with any $\epsilon>0$. This completes the proof of Theorem 1.1.

\section{ACKNOWLEGMENTS}

The author would like to thank Professor Jianya Liu and Professor Yangbo Ye for their constant encouragement and support. He is also grateful to the referee for valuable suggestions and detailed comments.

\section{REFERENCES}

[1] J. Conrey, D. Farmer, J. Keating, M. Rubinstein and N. Snaith, Integral moments of L-functions, Proc. London Math. Soc., (3) 91 (2005), 33-104. MR2149530 (2006j:11120)

[2] S. Gelbart and F. Shahidi, Boundedness of automorphic L-functions in vertical strips, J. Amer. Math. Soc. 14 (2001), 79-107. MR1800349(2003a:11056)

[3] R. Godement and H. Jacquet, Zeta functions of simple algebras, Lecture Notes in Math. 260, Springer-Verlag, Berlin, 1972. MR0342495 (49:7241) 
[4] D. R. Heath-Brown, Fractional moments of the Riemann zeta function, J. London Math. Soc. 24 (1981), No. 1, 65-78. MR623671 (82h:10052)

[5] H. Iwaniec and E. Kowalski, Analytic Number Theorem, Amer. Math. Soc. Colloquium Publ. 53, Amer. Math. Soc., Providence, RI, 2004. MR2061214 (2005h:11005)

[6] H. Jacquet and J. A. Shalika, A non-vanishing theorem for zeta functions of $G L_{n}$, Inventiones Math. 38 (1976), 1-16. MR0432596 (55:5583)

[7] J. Liu and Y. Ye, Superposition of zeros of distinct L-functions, Forum Math. 14 (2002), 419-455. MR.1899293 (2003g:11053)

[8] W. Luo, Z. Rudnick, and P. Sarnak, On the generalized Ramanujan conjecture for $G L(n)$, in Automorphic forms, automorphic representations and arithmetic, Proc. Symp. Pure Math. 66 (1999), Part 2, Amer. Math. Soc., Providence, RI, 301-310. MR1703764 (2000e:11072)

[9] G. Molteni, Upper and lower bounds at $s=1$ for certain Dirichlet series with Euler product, Duke Math. J. 111 (2002), 133-158. MR.1876443 (2002m:11084)

[10] K. Ramachandra, Some remarks on the mean value of the Riemann zeta-function and other Dirichlet series. I, Hardy-Ramanujan J. 1 (1978), 1-15. MR/565298(82f:10054a)

[11] K. Ramachandra, Some remarks on the mean value of the Riemann zeta-function and other Dirichlet series. II, Hardy-Ramanujan J. 3 (1980), 1-25. MR577338 (82f:10054b)

[12] Z. Rudnick and P. Sarnak, Zeros of principal L-functions and random matrix theory, Duke Math. J. 81 (1996), 269-322. MR1395406 (97f:11074)

[13] E. C. Titchmarsh, The theory of the Riemann zeta-function, 2nd Edition, The Clarendon Press, Oxford Univ. Press, New York, 1986. MR882550 (88c:11049)

Department of Mathematics, Shandong University, Jinan, Shandong 250100, People's Republic of China

E-mail address: ghji@mail.sdu.edu.cn 